\title{
Exploitation des eaux thermales Qualification de la ressource et stabilité des fluides
}

\author{
par Olivier Grière \\ de Geotherma SA
}

\section{I 圈 ASPECTS RÉGLEMENTAIRES}

\subsection{Définition d'une eau minérale}

La directive du Conseil de l'Union Européenne du 15 juillet 1980 définit dans son annexe le vocable « eau minérale naturelle » de la façon suivante:

- eau bactériologiquement saine c'est-à-dire présentant une teneur totale en micro-organismes revivifiables, à l'émergence, conforme à son microbisme normal et témoignant d'une protection efficace de la source contre toute contamination (absence de parasites, de micro-organismes pathogènes, absence d'Escherichia coli et autres coliformes dans $250 \mathrm{ml}$ à $37^{\circ} \mathrm{C}$ et $44,5^{\circ} \mathrm{C}$, absence de streptocoques fécaux dans $250 \mathrm{ml}$, absence d'anaérobies sporulés sulfito-réducteurs dans $50 \mathrm{ml}$, absence de Pseudomonas aeruginosa dans $250 \mathrm{ml}$ ),

- eau ayant pour origine une nappe ou un gisement souterrain et provenant d'une source exploitée par une ou plusieurs émergences.

L'eau minérale se distingue nettement de l'eau de boisson notamment :

- par sa nature, caractérisée par sa teneur en minéraux, oligo-éléments ou autres constituants et, le cas échéant par certains effets,

- par sa pureté originelle.

Une eau minérale naturelle ne peut faire l'objet d'aucun traitement ou adjonction à l'exception :

- de la séparation du fer à la condition que le process utilisé ne modifie pas la composition de cette eau dans ses constituant essentiels lui conférant ses propriétés,

\begin{abstract}
- de l'élimination totale ou partielle du gaz carbonique par des procédés exclusivement physiques,

- de l'incorporation ou la réincorporation de gaz carbonique dans des conditions strictes.

La législation française précise qu'une eau minérale doit présenter des propriétés favorables à la santé dûment constatées par l'Académie Nationale de Médecine.

Il faut remarquer qu'il n'existe pas de norme en terme de concentration pour les différents éléments physico-chimiques contrairement à une eau potable.
\end{abstract}

\subsection{Définition d'une eau thermale}

Il n'existe pas de définition précise pour qualifier une eau thermale.

Le décret 57-404 du 28 mars 1957 portant règlement d'administration publique sur la police et la surveillance des eaux minérales définit la procédure de demande d'autorisation d'exploitation.

Dans ce décret les eaux minérales et thermales sont regroupées. Pour obtenir l'autorisation d'exploitation, il est nécessaire, entre autre, de procéder à une analyse chimique et bactériologique de l'eau ainsi que des teneurs en gaz et de la radioactivité le cas échéant. Ce prélèvement doit être réalisé par un laboratoire agréé.

Ensuite l'autorité de tutelle fait procéder par le Laboratoire National de la Santé Publique à deux nouvelles analyses chimiques et bactériologiques à six mois d'intervalle. Enfin, une eau thermale doit présenter, tout comme pour une eau minérale, des propriétés thérapeutiques qui devront être reconnues.

After a short reminder on the reglementary basis which run the thermal and mineral waters exploitation in France, we will see the steps to undertake in order to qualify a new " spring ",

A thermal or mineral water is characterized by a specific physicochemical and bacteriological facies whose stability must be achieved.

We will examine through a few examples the inportance of the qualification conditions which must take into account the exploitation regime, the hydrogeological conditions and the specifities of each deposit. 


\subsection{Conclusion}

Il n'y a pas lieu de différencier les eaux minérales et thermales, si ce n'est dans l'utilisation de ces eaux. Les premières seront mises en bouteille et les secondes seront utilisées dans des établissements thermaux pour des soins divers et variés pouvant comporter des cures de boisson.

D'un point de vue qualitatif, ces eaux se caractérisent par une remarquable stabilité physico-chimique, une protection naturelle efficace et une qualité microbiologique irréprochable.

Il apparaît très clairement que ces eaux doivent donc présenter des caractéristiques constantes dans le temps dans des conditions d'exploitation clairement définies.

\section{QUALIFICATION D'UNE NOUVELLE «SOURCE »}

\subsection{Nature des informations à fournir}

Le demandeur doit élaborer un dossier complet pour déposer une demande d'exploitation. Ce dossier doit comporter, outre la localisation de l'ouvrage, le rapport géologique détaillé, la description technique du captage, un certain nombre d'examens portant sur la détermination :

- du débit.

- de la température de l'eau à l'émergence et de la température ambiante,

- des rapports existants entre la nature des terrains et la minéralisation,

- des résidus secs,

- de la conductivité (avec précision de la température de mesure)

- du pH, des anions et cations,

- des éléments non ionisés,

- des oligo-éléments.

- de la radio-actinologie à l'émergence,

- le cas échéant, des proportions relatives en isotopes des éléments constitutifs de l'eau $\left(\mathrm{O}^{18} \mathrm{O}^{16}\right.$, protium, deuterium, tritium),

- des caractéristiques microbiologiques.

Mais également, les eaux doivent faire l'objet d'examens cliniques et pharmacologiques.

\subsection{Conditions de prélèvement}

Le législateur s'est attaché à ce que les eaux minérales et thermales soient définies pour chacune d'entre elle, et ce quelque soit la grande variabilité des faciès physico-chimiques, par une stabilité remarquable et ce pour des conditions d'exploitation clairement définies. En effet, les eaux présentent des équilibres thermodynamiques fonction des conditions de gisement.

Lors de la mise en production d'un gisement, les équilibres initiaux peuvent être modifiés et engendrer des variations de composition.

De plus, dans le cas de gisements complexes où l'on peut assister à des mélanges naturels entre les formations il apparaît très clairement que les conditions de production (pompage) et les conditions hydrogéologiques (notamment piézométriques) influent sur les caractéristiques physico-chimiques voire microbiologiques.

A ce titre il faut remarquer que des émergences naturelles correspondent bien souvent à un mélange entre des eaux d'origine profonde ayant acquis une minéralisation avec des eaux météoriques peu minéralisées généralement.

La réalisation de nouveaux captages profonds ayant pour objectif le gisement thermal entraîne souvent la mise en évi- dence d'une eau dont les caractéristiques diffèrent de celles issues de l'émergence naturelle (température plus élevée, minéralisation plus importante...).

A l'émergence les eaux minérales ne sont pas en équilibre avec les conditions de surface, ceci peut se traduire par des dégagements de gaz, des dépôts de minéraux.

Les dépôts de minéraux sont d'importance variable et ils peuvent être constitués par de la silice, des carbonates de calcium (sources pétrifiantes), des oxydes de fer, des sulfates, des sulfures...

Ces phénomènes sont la manifestation de la néogenèse en profondeur des gîtes minéraux.

Ces dépôts hydrothermaux comblant les failles et fissures ont pu constituer des gisements exploitables d'un point de vue minier. Beaucoup de gisements exploités dans certaines régions se sont formés par des paléo-sources thermales, témoignages de l'instabilité des fluides.

\section{EXEMPLES}

\subsection{Eau thermale gazeuse}

\section{a) Considérations générales}

Geotherma a réalisé un forage profond dans le Massif Central dont les principales caractéristiques sont (cf. fig. l):

- profondeur : $500 \mathrm{~m}$,

- niveau capté : sables quartzo-feldspathiques et argiles bariolées anté Stampien,

- forage artésien.

De façon à mieux connaître le gisement diverses diagraphies différées ont été réalisées : Diamétreur, Polarisation Spontanée, Induction, Débitmétrie, Thermométrie.

Le micromoulinet a permis de localiser les arrivées d'eau qui se répartissent en deux zones distinctes de production quasi-équivalentes.

Un test de production (cf. fig. 2) avec observation de la remontée de pression a permis de déterminer la pression de gisement, le débit spécifique, la transmissivité et la porosité.

Ce puits produit une eau gazeuse par gaz-lift à une température à l'émergence de $75^{\circ} \mathrm{C}$ et un ratio gazleau de 2 lors de la mise en production.

Dans cet exemple, nous avons à faire à une eau de type bicarbonatée sodique gazeuse.

Nous avons donc un fluide qui se présente en tête de puits sous la forme diphasique.

Le gaz analysé est constitué à $98 \%$ de $\mathrm{CO}_{2}$ et $1,2 \%$ de $\mathrm{N}_{2}$.

\section{b) Implications}

La spécificité du fluide nécessitait de mieux appréhender le comportement du fluide à l'intérieur de l'ouvrage. En effet, les caractérisations physico-chimiques avaient été effectuées à l'émergence.

Il a donc été décidé de prélever des échantillons de fluide in situ pour préciser les caractéristiques du fluide.

- Principe de la mesure du point de bulle.

Geoservices (Société du groupe) a développé un analyseur sur site dénommé PVT 101B. L'échantillon d'eau prélevé dans le puits à une profondeur déterminée est transféré de la cellule d'échantillonnage à une cellule d'étude en maintenant la pression d'échantillonnage.

La cellule est ensuite montée à la température mesurée à la côte du prélèvement. 


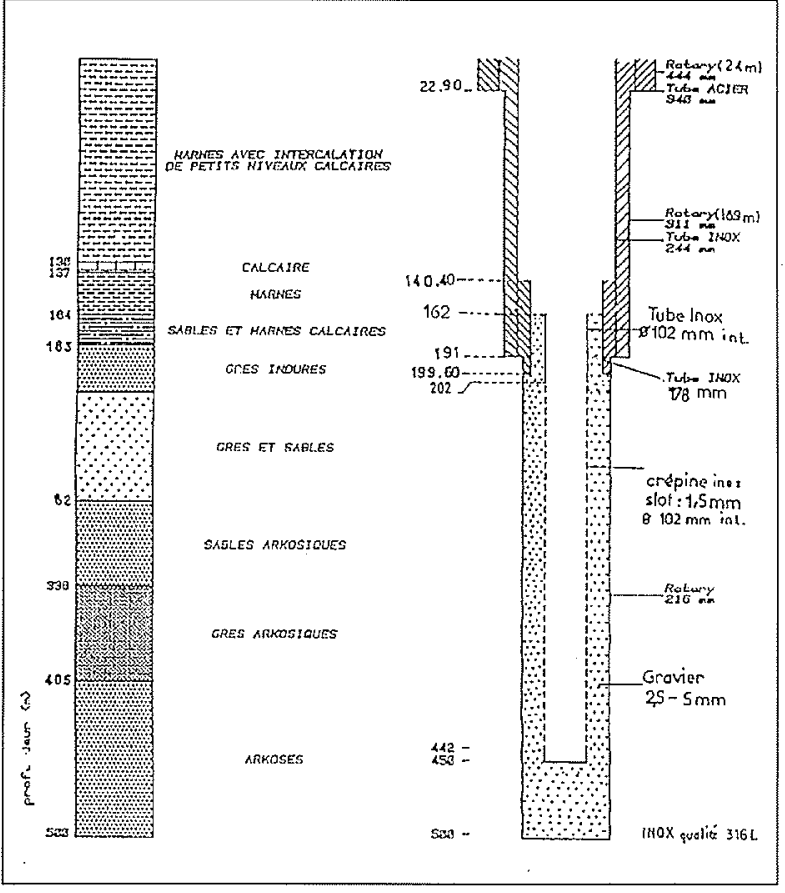

1. Coupe géologique et technique.

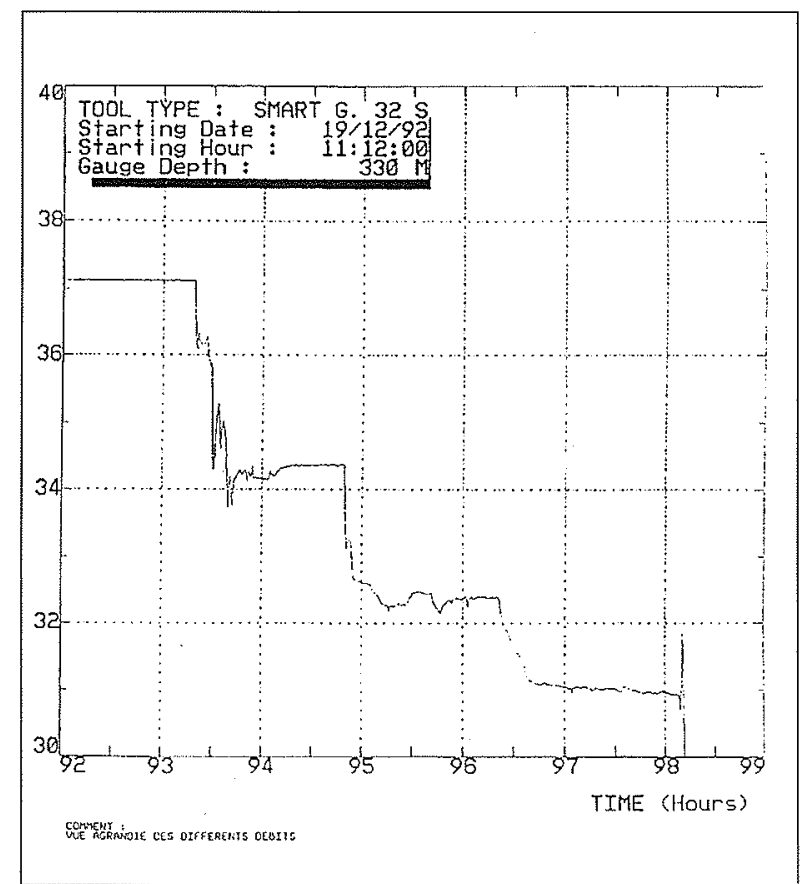

2. Test de production. Evolution de la pression dans l'ouvrage.

La pression est ensuite abaissée par mini-paliers. Tant que le fluide est monophasique, l'augmentation de volume est très faible, la compressibilité de la phase liquide variant très peu. A l'apparition de la première bulle, le fluide devient diphasique et la pression ne baisse plus et devient constante.

Les courbes volume relatif/pression et compressibilité/pression indiquent alors très précisément la valeur du point de bulle.

\section{c) Principaux résultats (cf. figs. 3 et 4 )}

Deux échantillons de fluide ont été prélevés à des profondeurs différentes et ont permis d'obtenir les résultats suivants :

\begin{tabular}{|c|c|c|}
\hline Profondeur & Point de bulle & Rapport gaz/eau \\
\hline $200 \mathrm{~m}$ & 22,8 bars & 3,7 \\
$330 \mathrm{~m}$ & 17,8 bars & 3,3 \\
\hline
\end{tabular}

Il apparaît donc que le comportement de débit de ces deux couches est apparemment très différent :

- la couche inférieure produit un fluide avec GWR (rapport eau/gaz) plus faible que la couche supérieure,

- la production naturelle doit s'effectuer en écoulement diphasique à partir du niveau de la couche supérieure.

D'autre part, l'interprétation hydrogéologique des tests de production confirme une production du type bicouche.

Ces constats montrent que le fluide lors de son ascension subit des modifications physiques qui doivent être prises en compte pour l'exploitation de l'ouvrage dans la mesure où un pompage serait envisagé.

\section{d) Conclusion}

Cet exemple caractéristique des gisements carbo-gazeux montre que la prise en considération des conditions hydro-
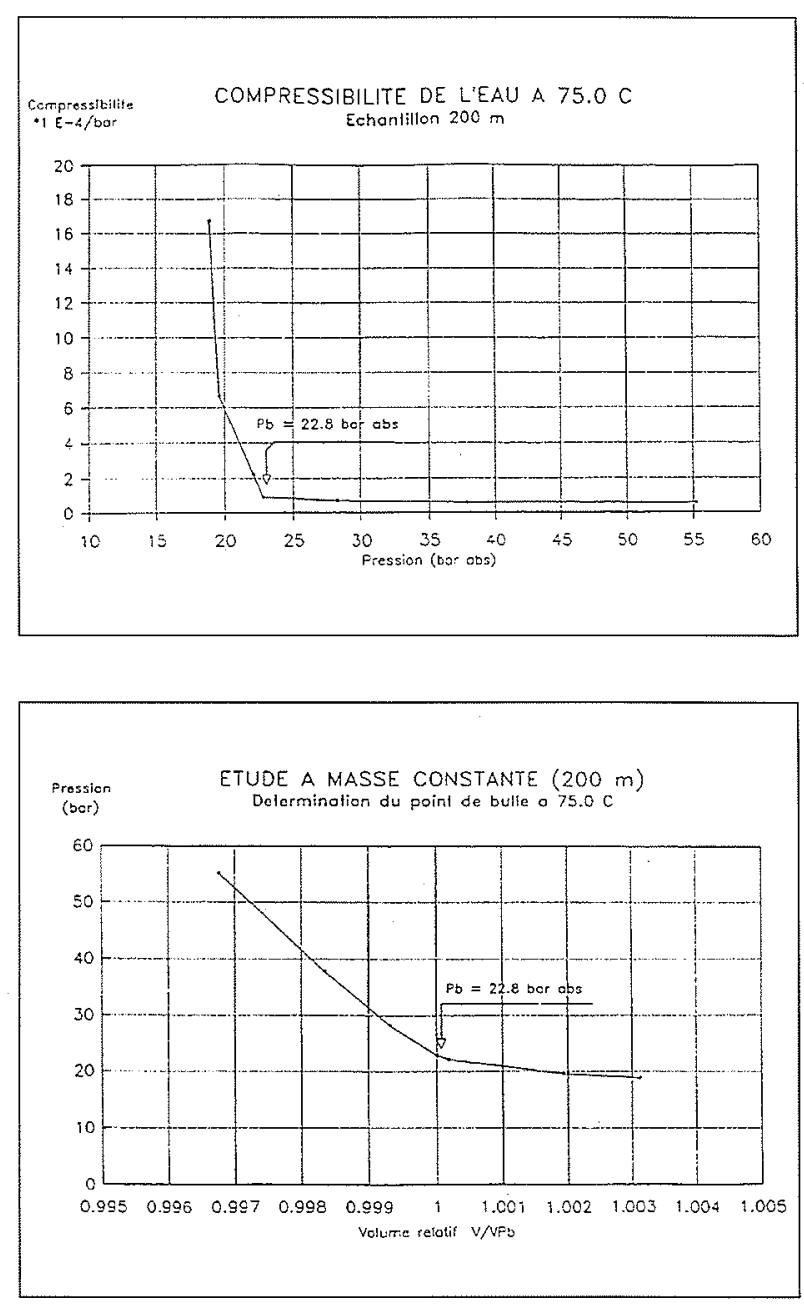
géologiques en vue de la détermination du régime d'exploitation est indispensable pour conserver au fluide des caractéristiques physico-chimiques constantes.

Des prélèvements en vue d'analyses effectués dans des conditions de production variable donneraient des résultats fluctuants en contradiction avec la notion de stabilité d'une eau thermale.

\subsection{Eau thermale sulfurée}

Les eaux sulfurées se caractérisent par une odeur particulière caractéristique due à la présence de $\mathrm{l}^{\prime} \mathrm{H}_{2} \mathrm{~S}$, mais également par une instabilité au contact de l'air.

Les espèces chimiques du soufre sont nombreuses. D'un point de vue thermodynamique les différentes espèces peuvent coexister soit par réaction d'équilibre $\mathrm{pH}$ ou d'oxydoréduction.

Sans entrer dans le détail, il convient de garder à l'esprit les principaux paramètres influençant les équilibres, il s'agit de :

- la température modifiant les équilibres thermodynamiques,

- la pression partielle en oxygène,

- la pression partielle en anhydride carbonique modifiant le $\mathrm{pH}$.

Il apparaît donc très clairement que dans le cas de gisements thermaux complexes présentant plusieurs arrivées d'eau distinctes (ce qui est souvent le cas) les conditions de pompage peuvent entraîner des modifications significatives de la minéralisation.
De plus, les conditions de prélèvement pour analyse doivent respecter un protocole très strict nécessitant les déterminations sur place.

Actuellement la méthode de référence est celle développée par Boulegue et Popoff.

\section{CONCLUSIONS}

Les fluides thermaux constituent des eaux spécifiques d'un point de vue minéralisation. Cette minéralisation spécifique à chaque gisement confère à cette eau des vertus thérapeutiques.

Ces eaux doivent présenter une grande stabilité dans le temps.

Nous avons vu que le régime d'exploitation, les conditions hydrogéologiques pouvaient modifier les équilibres. Il apparaît indispensable que chaque "source » soit connue très précisément tant d'un point de vue hydrogéologique (position des arrivées d'eau) que physico-chimique et bactériologique de chaque arrivée d'eau ainsi que d'un point de vue conditions d'exploitation (débit, rabattement, conditions piézométriques).

Une augmentation des débits pompés peut-être l'occasion de modifications significatives des caractéristiques du fluide.

Enfin, rappelons que les moyens d'analyses ont évolués et permettent aujourd'hui de disposer d'analyses fines avec des protocoles stricts et qu'il existe des outils spécifiques pour étudier les gisements (diagraphies, prélèvements in situ, tests en laboratoire...).

L'exploitation des eaux thermales passe immanquablement par des investigations fines et précises. 